\section{Nivolumab in NSCLC: latest evidence and clinical potential}

\author{
Raghav Sundar, Byoung-Chul Cho, Julie R. Brahmer and Ross A. Soo
}

Ther Adv Med Oncol 2015, Vol. 7(2) 85-96 DOI: $10.1177 /$ 1758834014567470

(c) The Author(s), 2015. Reprints and permissions: http://www.sagepub.co.uk/ journalsPermissions.nav

\begin{abstract}
New insight on the interaction between the immune system and tumor has identified the programmed death-1/programmed death-1 ligand pathway to be a key player in evading host immune response. The immune checkpoint modulator, nivolumab (BMS-936558/0NO4538), is the first PD-1 inhibitor to gain regulatory approval, for the treatment of patients with unresectable melanoma. This review will discuss results from early phase studies of nivolumab in solid tumors including non-small cell lung cancer (NSCLC) as well as studies of nivolumab in combination with chemotherapy, other immune modulators and molecular targeted therapy in patients with NSCLC.
\end{abstract}

Keywords: immune checkpoint modulator, nivolumab, non-small cell lung cancer, programmed death-1, programmed death-1 ligand

\section{Introduction}

A total of 1.6 million new cases of lung cancer are diagnosed each year, with 1.4 million deaths annually [Jemal et al. 2011]. Whilst advances in chemotherapy and molecular targeted therapy have improved outcomes in the advanced setting, survival remains poor with a 5 -year overall survival (OS) of 4-5\% [Howlader et al. 2014]. The advent of immunotherapy in non-small cell lung cancer (NSCLC) has begun to change the landscape of the management of this disease, offering a potential for prolonged responses and survival. For many years, lung cancer was believed to be nonimmunogenic with the failure of many agents such as Bacillus Calmette-Guerin (BCG), interleukin (IL)-2 and interferon [Einhorn et al. 1978; Jansen et al. 1992; Schiller et al. 1995]. However, new models of immunology and a better understanding of the interaction between the immune system and tumor has enabled the development of a new generation of cancer vaccines and immune modulators for NSCLC.

\section{Immune checkpoint inhibition}

Genetic and epigenetic variations in transformed cells lead to variation in antigen expression, but there is much controversy on whether antigenic signals on cancer cells are due to genomic instability of the cancer cells or associated with specific antigens related to the transformation [Schreiber et al.
2011; Wrangle et al. 2013]. Tumor recognition followed by tumor antigen presentation to $T$ cells and T-cell activation leads to tumor cell kill. T-cellmediated immune response is modulated by stimulatory and inhibitory signals. Checkpoint molecules (co-inhibitory molecules) include cytotoxic T-lymphocyte antigen-4 (CTLA-4), programmed death-1 (PD-1), T-cell immunoglobulin- and mucin domain-3-containing molecule 3 (TIM3), lymphocyte-activation gene 3 (LAG3) and killer cell immunoglobulin-like receptor (KIR) [Pardoll, 2012]. These immune checkpoints exist in a normal physiological state to protect against autoimmunity and inflammation. In a neoplastic state, dysfunction of these immune checkpoint proteins can lead to tumor tolerance and eventually allow for tumor 'escape' from the immune system. Targeting the molecules that regulate the immune response using antibodies has been the subject of much research and has yielded some promising and exciting results. This review will focus on a promising immune checkpoint inhibitor, an anti-PD-1 antibody, nivolumab. The role of other immune checkpoint inhibitors has been reviewed elsewhere [Brahmer, 2013, 2014; Brahmer and Pardoll, 2013; Sundar et al. 2014].

\section{PD-1 and PD-L1}

PD-1 receptor is expressed on several cells including CD4 and CD8 lymphocytes, B lymphocytes,
Correspondence to: Ross A. Soo, MBBS, FRACP Department of Haematology-Oncology, National University Cancer Institute, National University Health System, 1E Kent Ridge Road, NUHS Tower Block Level 7 , Singapore 119228 ross_soolanuhs.edu.sg Raghav Sundar, MBBS Department of Haematology-Oncology, National University Cancer Institute, National University Health System. Singapore

Byoung-Chul Cho, MD, PhD Division of Medical Oncology, Yonsei Cancer Center, Seoul, South Korea

Julie R. Brahmer, MD Department of Oncology, The Sidney Kimmel Comprehensive Cancer Center, Johns Hopkins, Baltimore, MD, USA 


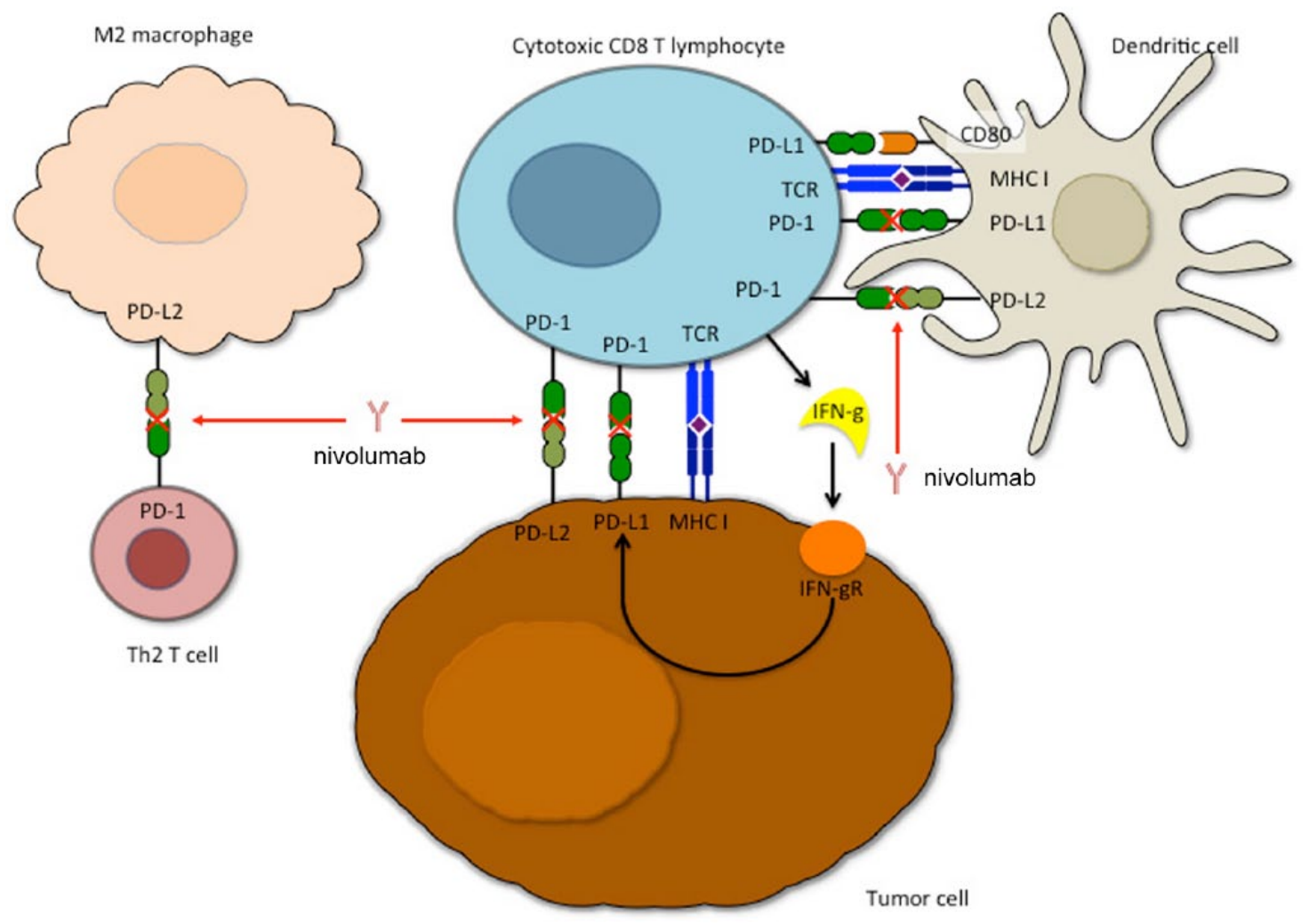

Figure 1. Interaction between PD-1/PD-L1 and T cell immune response. T cells are primed and activated through the interaction with antigen-presenting cells. T cells recognizes tumor via MHC-antigen interaction. Tumor PD-L1 and PD-L2 is upregulated by interferon $\gamma$ released from activated CD8 T cells. PD-L1 in turn inhibits T cells via PD-1.

IFN-g, interferon $\gamma$; IFN-gR, interferon $\gamma$ receptor; MHC-I, major histocompatibility complex class I; PD-1, programmed death-1; PD-2; programmed death-2; PD-L1, programmed death ligand-1; PD-L2, programmed death ligand-2; TCR, T cell receptor; Th, T helper.

natural killer (NK) cells and $\mathrm{T}$ regulatory cells (Tregs) [Keir et al. 2008]. Ligands of PD-1 include PD-L1 (or CD274, B7-H1) and PD-L2 (CD 273, B7-DC) [Dong et al. 2002]. PD-L1 is expressed in $\mathrm{T}$ and $\mathrm{B}$ cells, dendritic cells and macrophages (Figure 1). It is upregulated in a range of solid tumors including NSCLC. Upon induction by cytokines such as IL-4, IL-10, interferon (IFN) $\alpha, \beta$ or $\gamma$, PD-L1 activates PD- 1 on T cells, and downregulates T-cell effector function. Through this mechanism the PD-1 pathway plays a role in the control of T-cell activity in peripheral tissue during an inflammatory response. In a similar manner, tumor cells can exploit the PD-1/ PD-L1 pathway as a means of evading T-cellregulated immune surveillance. $\mathrm{PD}-\mathrm{L} 1$ is upregulated by IFN $\gamma$ produced by $\mathrm{T}$ cells or through constitutive oncogenic signaling via the mitogenactivated protein kinase (MAPK) pathway or the phosphoinositide 3-kinase (PIK3) pathways [Pardoll, 2012]. Constitutive signaling through the EGFR pathway can also promote tumor escape via activation the PD-1/PD-L1 pathway [Akbay et al. 2013].

PD-L1 is localized in the cell membrane and/or the cytoplasm and is expressed in about $20-65 \%$ of NSCLC [Konishi et al. 2004; Mu et al. 2011; Chen et al. 2012, 2013; Sundar et al. 2014]. The overexpression of PD-L1 in resected NSCLC is associated with a poorer prognosis [Chen et al. 2012; Azuma et al. 2014] whilst other studies have reported either improved outcomes [Velcheti et al. 2014] or no association with survival [Yang et al. 2014; Boland et al. 2013; Konishi et al. 2004]. In advanced stage NSCLC, no association between PD-L1 expression and survival was reported [Sorenson et al. 2014].

PD-L2 interacts with PD-1 to inhibit T-cell effector function [Tseng et al. 2001]. Unlike PD-L1, which is expressed more broadly, the expression 
of PD-L2 is more limited, with expression mainly in macrophages and dendritic cells [Rozali et al. 2012]. This suggests PD-L2 is less effective in regulating peripheral T-cell response [Chen et al. 2012]. The expression of PD-L2 in tumor tissue is associated with a poorer survival in patients with resected NSCLC [Zhang et al. 2014].

Several antibodies directed against PD-1 have been developed including nivolumab (BMS936558, Opdivo ${ }^{\circledR}$ ), pembrolizumab (MK3475, lambrolizumab, Keytruda ${ }^{\circledR}$ ), and pidilizumab (CT-011). AMP-224 is a fusion protein targeting PD-1. Nivolumab is the first PD-1 inhibitor to gain regulatory approval and is now approved for use in patients with unresectable melanoma in Japan [ONO Pharmaceutical, 2014]. Nivolumab has been granted Fast Track designation by the US FDA in NSCLC, melanoma and renal cell carcinoma. A rolling submission for nivolumab in the third-line treatment of squamous NSCLC was initiated in April 2014 based on the CheckMate063 study (to be discussed later) and submission is expected to be completed by the end of 2014 [Bristol-Myers Squibb, 2014]. In this review we present the latest data on nivolumab in NSCLC.

\section{Nivolumab}

Nivolumab/MDX-1 106/BMS-936558/ONO4538 is a genetically engineered, fully human immunoglobulinG4 (IgG4) monoclonal antibody specific for human PD-1. The IgG4 isotype was engineered to obviate antibody-dependent cellular cytotoxicity (ADCC). Most monoclonal antibodies in therapeutic oncology contain the IgG1 subtype, which have the most significant ADCC whereas IgG4 subtype possesses minimal ADCC activity. An intact ADCC has the potential to deplete activated $\mathrm{T}$ cells and tumor-infiltrating lymphocytes and diminish activity as PD-1 is expressed on $\mathrm{T}$ effector cells and other immune cells [Chen et al. 2012]. Nivolumab binds PD-1 with high affinity (KD $2.6 \mathrm{nmol} / 1$ by Scatchard analysis to polyclonally activated human $\mathrm{T}$ cells) and blocks its interactions with both $\mathrm{B} 7-\mathrm{H} 1$ and B7-DC [Brahmer et al. 2012].

\section{Early phase trials of nivolumab in lung cancer}

In a first in human phase I study, patients with advanced stage solid tumors were treated with a single dose of nivolumab at $0.3,1,3$ or 10 $\mathrm{mg} / \mathrm{kg}$ [Brahmer et al. 2010]. Patients with clinical benefit were eligible for repeat dosing at 3 months. Nivolumab was well tolerated, dose-limiting toxicities (DLTs) were not reached and the maximum tolerable dose (MTD) was not defined. Of the six patients with NSCLC, one had significant lesional response not amounting to a partial response (PR). Response was seen in 12 out of the 39 patients. Whilst the measured half-life of nivolumab was $12-20$ days, the pharmacodynamic effects of PD-1 receptor occupancy was even more prolonged at 85 days, indicating the biological durability of this high-affinity $\mathrm{mAb}$. Based on the safe toxicity profile and signs of clinical activity, a larger multidose trial was subsequently conducted.

In a phase Ib study of patients with advanced stage solid tumors including melanoma, NSCLC, renal cell cancer, prostate cancer and colorectal cancer, escalating doses of nivolumab biweekly were administered [Topalian et al. 2012]. Of the NSCLC cohort $(n=129)$ many were heavily pretreated, with $54 \%$ receiving at least 3 prior lines of therapy. The overall response rate (ORR) was $17 \%$ with a median duration of response of 74 weeks (range 6.1-133.9 weeks). Amongst the responders, $50 \%$ showed reduction in tumor size within the first 2 months of treatment. $33 \%$ of the squamous tumors ( 6 out of 18 ) and $12 \%$ of the nonsquamous tumors (7 out of 56) responded. Sustained response of $>24$ weeks was seen in $57 \%(8 / 14)$ of patients and 2 patients had a response of more than 1 year. Stable disease of more than 24 weeks was seen in 5 of the nonsquamous tumors. Updated results presented in abstract form reported prolonged survival with a median of 9.2-14.9 months across the cohorts with a 1-year survival of $32-56 \%$ and $12-45 \%$, respectively. For the 3 $\mathrm{mg} / \mathrm{kg}$ cohort, the median OS was 14.9 months, 1 -year OS of $56 \%$ and 2 -year OS of $45 \%$ [Brahmer et al. 2014].

\section{Toxicity of nivolumab}

In the phase Ib study discussed previously, nivolumab was generally well tolerated, with $41 \%$ developing adverse events, but only $6 \%$ developing grade 3 or 4 toxicities. Skin toxicities were the most common toxicity occurring in $31 \%$ of patients, with the most common being that of rash (12\%), pruritus (9\%) and vitiligo (3\%). Gastrointestinal toxicities such as diarrhea 
occurred in about $11 \%$, while pneumonitis occurred only in $3 \%$ of patients, with only $1 \%$ having grade 3 or 4 pneumonitis. Other toxicities included abnormalities in transaminases (7\%), thyroid dysfunction (3\%) and infusion-related reactions $(3 \%)$.

Pneumonitis is a serious adverse event and is of major concern in lung cancer patients who may already have poor lung reserve due to prior smoking or metastatic disease. In the lung cancer cohort, pneumonitis was reported in $6 \%$ (8/129), $2 \%$ was grade $3 / 4$ (3 patients) and two patient deaths were attributed to pneumonitis [Brahmer et al. 2013]. These toxicities have been similar to other PD-1 agents and other nivolumab trials [Brahmer et al. 2012; Horn et al. 2013; Garon et al. 2014b]. Pneumonitis rates for nivolumab are similar to or lower than rates of other commonly used drugs in NSCLC such as docetaxel (4.6\%) [Grande et al. 2007] and gefitinib (3.5\%) [Konishi et al. 2005]. However, as nivolumab is currently being tested in combination with several other agents, cumulative toxicity of pneumonitis would be of concern. This is of particular concern since several patients with advanced NSCLC are likely to have poor lung reserve function and prior thoracic radiation might also worsen pulmonary inflammation and pneumonitis.

PD-L1 is expressed in the small intestine [Keir et al. 2008] whereas PD-L2 is important in regulating TH2-mediated inflammation in the lung [Akbari et al. 2010]. PD-1 inhibitors are intended to block PD-1 from interacting with any of its ligands, hence preventing engagement between PD-1 with PD-L1 and PD-1 with PD-L2. In comparison PD-L1 inhibitors prevent binding between PD-1 with PD-L1 and PD-L1 with CD80 (B7.1) but not necessarily PD-1 with PD-L2 binding. The different mechanism of action in agents targeting PD-1 and PD-L1 may potentially result in a different sideeffect profile. PD-L1 inhibitors may potentially have a lower risk of pneumonitis whereas targeting PD-1 may have a lower risk of gastrointestinal side effects. As the studies conducted to date differ greatly in terms of patient population, sample size, and duration of follow-up, direct comparisons between the PD-1 and PD-L1 inhibitors should be made with great caution. Further studies are required to determine differences in side effects between PD-1 and PD-L1 inhibitors.

\section{Current ongoing trials of nivolumab in lung cancer}

There are currently multiple trials examining the efficacy of nivolumab in patients with advanced stage NSCLC (Table 1). Of interest is the combination of PD-1 blockade with CTLA-4 inhibition (nivolumab plus ipilimumab) and combination with other immune checkpoint modulators. In preclinical studies multiple immune checkpoint blockades with combination PD-1 and CTLA4 $\mathrm{Ab}$ can allow for increased T-cell responsiveness and decreased T-cell anergy, in preclinical models [Curran et al. 2010]. This approach has been supported by results of a phase I study of nivolumab and ipilimumab in patients with advanced-stage melanoma where an ORR of $53 \%$ was observed in patients receiving concurrent nivolumab and ipilimumab [Wolchok et al. 2013]. However, a cautious approach is warranted given the potential to exacerbate autoimmunity.

Cytotoxic chemotherapy, targeted therapies and radiotherapy can modulate immune response to tumors. An understanding of these immunomodulatory effects may enable the design of rational combinations of chemotherapy, targeted therapy and radiotherapy with immunotherapy. Cytotoxic chemotherapy can modulate the immune system by several mechanisms such as: (1) inducing immunogenic cell death, a form of cell death that induces dendritic cells to stimulate tumor antigen presentation to $T$ cells [Kroemer et al. 2013]; (2) stimulating T-cell activation via increasing the expression of MHC-1 molecules (such as oxaliplatin, gemcitabine, paclitaxel) [Liu et al. 2010]; (3) stimulating dendritic cell maturation (gemcitabine, oxaliplatin) [Liu et al. 2010]; and (4) reducing the immunosuppressive function of regulatory $\mathrm{T}$ cells (cyclophosphamide, paclitaxel) [Ghiringhelli et al. 2007; Zhang et al. 2008] and myeloid derived suppressor cells (MDSCs; gemcitabine, docetaxel) [Suzuki et al. 2005; Kodumudi et al. 2010]. Vascular endothelial growth factor (VEGF) may have immunosuppressive effects. VEGF stimulate MDSC in peripheral immune organs, promote regulatory $\mathrm{T}$ cells and inhibit dendritic cell maturation [Goel et al. 2011]. As such VEGF inhibition in combination with a checkpoint inhibitor may be synergistic [Huang et al. 2013] and a study using this combination in advanced stage NSCLC is ongoing [Clinical Trials. gov identifier: NCT01454102]. Ionizing radiation can increase tumor susceptibility to T-cell killing through increased expression of death receptors, MHC class 1 molecules, costimulatory 


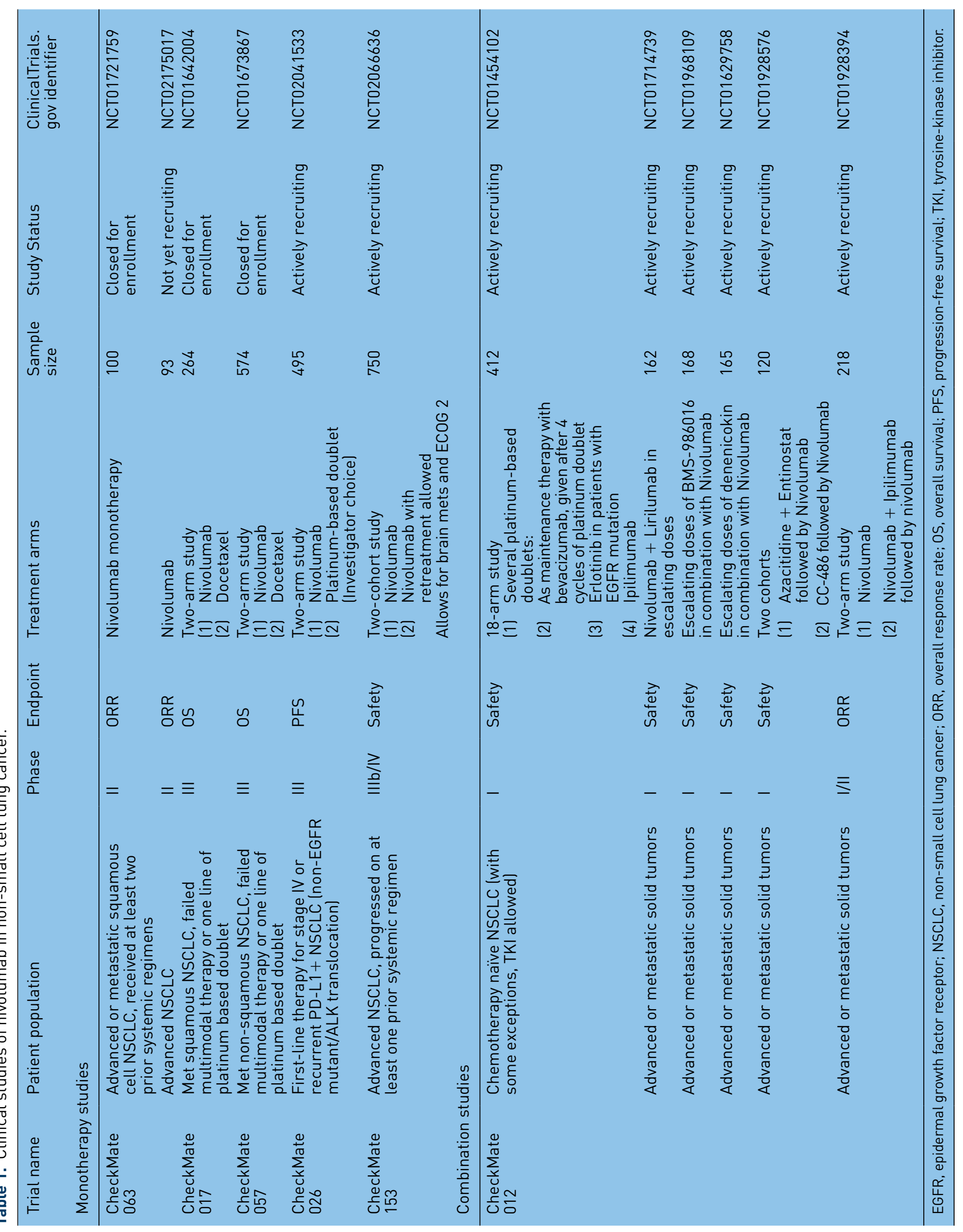


Table 2. Preliminary results from the multi-arm phase I study of nivolumab (Checkmate 012).

\begin{tabular}{|c|c|c|c|c|c|c|c|}
\hline Study & $N$ & Patient population & Treatment & ORR & 24 week PFS & $\begin{array}{l}\text { Median OS } \\
\text { (weeks) }\end{array}$ & $\begin{array}{l}1 \text {-year } \\
\text { survival }\end{array}$ \\
\hline $\begin{array}{l}\text { Antonia et al. } \\
\text { [2014] }\end{array}$ & 56 & Chemonaïve & $\begin{array}{l}\text { Nivolumab } \\
+ \text { platinum } \\
\text { doublet }\end{array}$ & $33-47 \%$ & $38-71 \%$ & $50.5-83.4$ & $50-87 \%$ \\
\hline $\begin{array}{l}\text { Rizvi et al. } \\
\text { [2014] }\end{array}$ & 21 & $\begin{array}{l}\text { Chemonaïve, EGFR } \\
\text { mt positive }\end{array}$ & $\begin{array}{l}\text { Nivolumab }+ \\
\text { erlotinib }\end{array}$ & $19 \%$ & $51 \%$ & NR & $73 \%$ \\
\hline $\begin{array}{l}\text { Gettinger et al. } \\
\text { [2014] }\end{array}$ & 20 & Chemonaïve & Nivolumab & $\begin{array}{l}22 \% \text { SQ } \\
36 \% \text { NSQ } \\
50 \% \text { PDL1+ }\end{array}$ & $\begin{array}{l}\text { 70\% PDL1+ } \\
57 \% \text { PDL1 - }\end{array}$ & NR & $71-80 \%$ \\
\hline $\begin{array}{l}\text { Antonia et al. } \\
\text { [2014] }\end{array}$ & 49 & Chemonaïve & $\begin{array}{l}\text { Nivolumab }+ \\
\text { ipilimumab }\end{array}$ & $\begin{array}{l}\text { 14\% PDL1-19\% } \\
\text { PDL1+ }\end{array}$ & $\begin{array}{l}25 \% \text { SQ } \\
51 \% \text { NSQ }\end{array}$ & 44.3 & NR \\
\hline
\end{tabular}

molecules, adhesion molecules and stress-induced ligands [Formenti and Demaria, 2013, Frey et al. $2014]$. As these anticancer treatments can potentially modulate antitumor immune response, there is good rationale to combine it with immune checkpoint inhibitors.

As immune checkpoint inhibitors induce T-cell response, patients treated with supraphysiological doses of corticosteroids are inappropriate candidates for immune checkpoint inhibitors as systemic corticosteroids may attenuate the potential beneficial effects of these drugs [Harvey, 2014]. As such, patients on systemic doses of corticosteroids have been excluded from clinical trials of nivolumab and other immune checkpoint inhibitors. [Topalian et al. 2012; Brahmer et al. 2012; Wolchok et al. 2013; Hamid et al. 2013]. Low-dose steroids as an anti-emetic or premedication for paclitaxel [Lynch et al. 2012] can be used, as it is unlikely that the therapeutic effect of immune checkpoint inhibitors will be affected at low doses. In fact, continued antitumor activity has been observed in patients with immune-related adverse events treated with highdose steroids [Downey et al. 2007; Harmankaya et al. 2011]. Generally steroids may be used if needed, but should be avoided if not required [Fecher et al. 2013].

CheckMate 012 [ClinicalTrials.gov identifier: NCT01454102] is a multi-arm phase I study of nivolumab with various anticancer agents or as monotherapy (Table 1). Preliminary results from four treatment arms from this study have been presented recently at the 2014 ASCO and are summarized below (Table 2).
In the platinum doublet arm, where three platinumbased chemotherapy regimens (cisplatin/gemcitabine; cisplatin/pemetrexed; and carboplatin/ paclitaxel) were combined with nivolumab $(n=56)$, the ORR was 33-50\%, 24-week progression-free survival (PFS) $36-71 \%$ and 1-year OS was $59-87 \%$ [Antonia et al. 2014a]. No DLTs were seen in the first 6 weeks of treatment, but $45 \%$ of patients had grade 3 or grade 4 toxicities and $7 \%(n=4)$ had pneumonitis. Based on these preliminary results, the antitumor activity of first-line nivolumab in combination with platinum doublets is highly promising.

In the erlotinib and nivolumab arm, chemotherapy-naïve patients $(n=21)$ with $E G F R$ mutations were enrolled. A total of 20 patients had prior treatment with first-line erlotinib. The ORR was $19 \%$ and the 24 -week PFS was $51 \%$ [Rizvi et al. 2014]. Interestingly, of those with acquired erlotinib resistance, 3 (15\%) had a PR, $9(45 \%)$ achieved SD, and 1 had an unconventional immune related response. Grade 3 toxicities were reported in $19 \%$ of patients (there were no grade 4 toxicities). Common side effects included skin rash, fatigue, paronychia, diarrhea and skin fissures. Whilst this combination show encouraging activity, the data is preliminary and validation and comparison with the third-generation EGFR tyrosine-kinase inhibitors (TKIs) in tumors harboring T790M mutations would be needed. Further work on the interaction between tumors harboring $\mathrm{T} 790 \mathrm{M}$ mutations and the tumor microenvironment and immune checkpoints would also be of interest.

In the cohort of chemotherapy-naïve patients with advanced NSCLC treated with single-agent 
nivolumab $(n=20)$, the ORR was $30 \%$. The 1 -year OS and median PFS was $75 \%$ and 36 weeks, respectively [Gettinger et al. 2014]. Grade 3/4 treatment related toxicities were reported in four $(20 \%)$ patients. In exploratory analysis, the ORR, PFS rate at 24 weeks, median $\mathrm{PFS}$ and 1-year OS were numerically higher in patients with tumors expressing PD-L1 compared with PD-L1 negative tumors. A phase III study of single-agent nivolumab versus investigator choice of a platinum doublet in the first-line treatment of advanced stage NSCLC is ongoing [ClinicalTrials.gov identifier: NCT02041533].

In the nivolumab and ipilimumab combination arm, the ORR was $22 \%$, stable disease was $33 \%$ and the PFS rate at 24 weeks was $20-51 \%$. No association was found between PD-L1 status and clinical activity. Treatment-related adverse events were reported in $88 \%$ of patients, most commonly fatigue $(45 \%)$. Pneumonitis was reported in six $(12 \%)$ patients (all grades) and three $(6 \%)$ were grade 3/4. All cases were reversible upon discontinuation of study drugs and use of corticosteroids. Grade 3-4 treatment-related toxicity was reported in 24 patients (49\%). Diarrhea was the most common toxicity (10\%) [Antonia et al. 2014b] Treatment-related deaths occurred in three patients (two from pulmonary toxicities and one from toxic epidermal necrolysis). The data, although preliminary, is promising as this combination is active in both PD-L1-positive and PD-L1-negative NSCLC. This combination may be warranted in selected patients, such as patients with PD-L1-negative tumors where nivolumab alone may have lower clinical activity. Additional studies are required to further define safety and clinical activity of this combination.

Checkmate 063 (Table 1) is a study of singleagent nivolumab in patients who had failed two or more lines of systemic therapy. Preliminary results reported an ORR of $15 \%$ (95\% confidence interval [CI] 8.7-22.2), with a median OS of 8.2 months and a 1 -year OS of $41 \%(95 \%$ CI 31.6-49.7). Pneumonitis rate was reported as 3.4\% [Ramalingam et al. 2014]. Results are highly promising given $65 \%$ had been treated with three or more lines of therapy.

KIR is a family of NK-cell regulators represents a novel class of immunotherapy and is beginning to garner interest in various tumor types. KIR is one of the negative regulators of NK-cell effector function. The inhibition of KIR results in
NK-cell-mediated antitumor activity [Ruggeri et al. 2002]. Lirilumab is an antibody that binds to KIR2DL1, 2 and 3 receptors, causing NK-cellmediated cell kill [Romagne et al. 2009]. A phase I study combining lirilumab and nivolumab in patients with advanced tumors is ongoing [ClinicalTrials.gov identifier: NCT01714739].

Lymphocyte-activation gene 3 (LAG3; CD223) is a co-inhibitory receptor expressed in activated T cells, Tregs, dendritic cells and NK cells. LAG3 is a CD4-related protein that binds to major histocompatibility complex (MHC) class II and reduces T-cell proliferation resulting in tumor evasion [Huang et al. 2004; Gandhi et al. 2006]. LAG3 gene expression is upregulated in a silicamediated lung inflammation murine model [Freire et al. 2013]. Further work is needed to characterize LAG3 in NSCLC, with a current phase 1 study looking at the role of BMS-986016, a LAG3 monoclonal antibody with or without nivolumab in advanced solid tumors [ClinicalTrials.gov identifier: NCT01968109].

The role of epigenetic priming on subsequent treatment with a PD-1 checkpoint inhibitor is being investigated. It is hypothesized that epigenetic modulation with azacitidine, a cytidine analogue that causes DNA hypomethylation, and entinostat, a histone deacetylase inhibitor, affects inflammation within the tumor by affecting the interferon pathway, leading to PD-L1 upregulation. Azacitidine can also induce cancer testis antigens which are not expressed in normal tissues (except the testes in males and the placenta in pregnant females), while being expressed almost 'tumor-specifically' in several different tumors [Van Den Eynde and Boon, 1997]. This leads to an enhanced immune response against the tumor. A study of azacitidine and entinostat or azacitidine alone prior to nivolumab with the primary endpoint of ORR is ongoing [ClinicalTrials.gov identifier: NCT01928576].

\section{Comparison with other PD-1/PD-L1 inhibitors}

Although cross-trial comparisons are not possible, especially in this very early stage of development of various immune checkpoint inhibitors, PD-1/PD-L1 inhibitors appear to have similar efficacy in pretreated advanced stage NSCLC. The ORR of the anti-PD-1 antibody pembrolizumab (MK-3475) is 15-24\% [Garon et al. 2013, 2014a]. In a pooled analysis of pembrolizumab in 
phase I studies, an ORR of $21 \%(16-27 \%)$ and a median PFS of 27 weeks (95\% CI 14-45 weeks) was reported in treatment-naïve patients, with a 24 -week PFS of $51 \%$. In pretreated patients, the median PFS was 10 weeks [Garon et al. 2014a]. Anti-PD-L1 antibodies MPDL3280A and MEDI4736 have ORR of $23 \%$ and $15 \%$, respectively [Soria et al. 2013, Lutzky et al. 2014], similar to that of nivolumab with an ORR of $24.3 \%$ (3mg/kg cohort) [Brahmer et al. 2014b].

Toxicities amongst various antibodies of the same class appear similar as well, with most drugs reporting a pneumonitis rate of less than $5 \%$, while the most common adverse event is that of fatigue. Grade 3 or 4 toxicities of all adverse events appear to be less than $10 \%$ for all drugs, suggesting that this class of drugs is a very well tolerated.

\section{Measurement of tumor response}

One of the challenges faced in the development of nivolumab and other inhibitors of the PD-1/ PD-L1 pathway is the assessment of tumor response. The use of RECIST1.1 for tumor assessment in patients receiving immunotherapy has limitations. For example, it does not capture (i) patients who initially progress as defined by RECIST 1.1 but subsequently respond or (ii) patients with a mixed response or new lesions, but the overall tumor burden is decreased. Based on this, an immune-related response criterion has been created [Wolchok et al. 2009]. Immunerelated progression-free survival (irPFS) accounts for the apparent increase in tumor size followed by sustained tumor response, which has been documented with these agents in the past [Oxnard et al. 2012]. This phenomenon of 'pseudo-progression' may be due to peritumoral lymphocyte infiltration or delayed immune activity.

\section{Biomarkers}

The need for predictive biomarkers is important, as nivolumab is highly active in a very select group of patients. In the landmark phase I study, the association between ORR and PD-L1 expression in NSCLC was investigated [Topalian et al. 2012]. PD-L1-positive tumors, defined as expression in at least $5 \%$ of tumor cells on immunohistochemistry (IHC), were seen in 49\% (31/63) of patients. The ORR in these heavily pretreated patients with PD-L1 positive and PD-L1 negative tumors was $16 \%$ and $13 \%$, respectively [Antonia et al. 2013]. Using archival tumor tissue may not be ideal for assessing PD-L1 status especially in a heavily pretreated group of patients hence the disparate results between studies. In a more recent study of chemotherapy-naïve patients with advanced-stage NSCLC treated with nivolumab, the ORR was $50 \%$ in PD-L1-positive tumors whereas the ORR was $0 \%$ in PD-L1-negative tumors [Gettinger et al. 2014]. The use archival tumor samples from pretreated patients in the pivotal phase I study may explain the differences in ORR according to PD-L1 expression between the two studies. PD-L1 expression appeared to correlate with pembrolizumab ORR, with strong PD-L1 expression ( $>50 \%$ ) having an ORR of $39 \%$, while PD-L1 negative being only $9 \%$ [Garon et al. 2014a]

As the frequency of PD-L1 positive NSCLC tumors is about $20 \%$ [Sundar et al. 2014], potentially a large number of patients with advanced stage NSCLC may be suitable for nivolumab treatment. In comparison, the frequency of patients with EML-ALK translocation or ROS1 rearrangement is about 4\% [Solomon et al. 2009] and 1-2\% [Leow et al. 2012], respectively, depending on the population studied and detection methods used.

It is recognized PD-L1 expression varies with the tumor microenvironment [Taube et al. 2012] and thus PD-L1 expression at a single time point may not represent a dynamic immune response. Thus PD-L1 expression as a predictive biomarker may be difficult to implement. Further studies are needed to characterize immunologic correlates of response in the pretreatment and on-treatment setting.

\section{Future directions}

Several questions regarding the optimal duration of therapy and the role of retreatment with nivolumab are yet to be answered. A recent clinical trial [ClinicalTrials.gov identifier: NCT02066636] described aims to answer some of these questions. Issues of long-term toxicities and consequences of prolonged exposure to immunotherapy are to be addressed by this study.

It has been reported that tumor bulk can affect the ability to mount a successful tumor-specific immune response [Stewart and Abrams, 2007]. One potential approach would be to administer nivolumab and other PD-1, PD-L1 inhibitors after complete gross tumor resection to target minimal residual disease. As such studies of nivolumab in the adjuvant setting should be explored. 
Another area of great interest is elucidating the interaction between oncogene-driven signaling pathways regulating immune escape and the regulation of tumor-infiltrating lymphocytes. It is likely that tumor genomic profiling will be integrated with profiling of the tumor microenvironment to provide a more comprehensive approach to treatment selection.

\section{Conclusion}

A deeper understanding of the immune system in tumor immunosurveillance has led to the development of a novel approach to the management of advanced stage NSCLC. Whilst results from early phase studies of immune checkpoint modulators such as nivolumab, in a range of solid tumors including NSCLC are highly promising, these trials require confirmation. Research is ongoing to combine immunotherapy agents with chemotherapy and molecular targeted therapy and to identify patients who are likely to respond to PD-1/ PD-L1-inhibitors.

\section{Acknowledgements}

RAS is supported by the National Research Foundation, Singapore and the Singapore Ministry of Education under its Research Centres of Excellence initiative.

\section{Conflict of interest statement}

RS, BCC and RAS have no conflicts of interest to declare. JBR has received research funding from Merck and BMS, is a paid consultant for Merck and is a compensated advisory board member for BMS.

\section{References}

Akbari, O., Stock, P., Singh, A., Lombardi, V., Lee, W., Freeman, G. et al. (2010) PD-L1 and PD-L2 modulate airway inflammation and iNKTcell dependent airway hyperreactivity in opposing directions. Mucosal Immunol 3: 81-91.

Akbay, E., Koyama, S., Carretero, J., Altabef, A., Tchaicha, J., Christensen, C. et al. (2013) Activation of the PD-1 pathway contributes to immune escape in EGFR-driven lung tumors. Cancer Discov 3: 13551363.

Antonia, S., Grosso, J. and Horak, C. (2013) Association of tumor PD-L1 expression and immune biomarkers with clinical activity in patients with non-small cell lung cancer treated with nivolumab. In:
IASLC 15th World Conference on Lung Cancer, Sydney, Australia.

Antonia, S., Brahmer, J., Gettinger, S., Chow, L., Juergens, R., Shepherd, F. et al. (2014a)

Nivolumab (anti-PD-1; BMS-936558, ONO4538) in combination with platinum- based doublet chemotherapy (Pt-DC) in advanced non-small cell lung cancer (NSCLC). ASCO Meeting Abstracts 32: 8113.

Antonia, S., Gettinger, S., Chow, L., Juergens, R., Borghaei, H., Shen, Y. et al. (2014b) Nivolumab (anti-PD-1; BMS-936558, ONO-4538) and ipilimumab in first-line NSCLC: interim phase I results. ASCO Meeting Abstracts 32: 8023.

Azuma, K., Ota, K., Kawahara, A., Hattori, S., Iwama, E., Harada, T. et al. (2014) Association of PD-L1 overexpression with activating EGFR mutations in surgically resected non-small cell lung cancer. Ann Oncol 25: 1935-1940.

Boland, J., Kwon, E., Harrington, S., Wampfler, J., Tang, H., Yang, P. et al. (2013) Tumor B7-H1 and B7-H3 expression in squamous cell carcinoma of the lung. Clin Lung Cancer 14: 157-163.

Brahmer, J. (2013) Harnessing the immune system for the treatment of non-small-cell lung cancer. $\mathcal{F}$ Clin Oncol 31: 1021-1028.

Brahmer, J. (2014) Immune checkpoint blockade: the hope for immunotherapy as a treatment of lung cancer? Semin Oncol 41: 126-132.

Brahmer, J., Drake, C., Wollner, I., Powderly, J., Picus, J., Sharfman, W. et al. (2010) Phase I study of single-agent anti-programmed death-1 (MDX-1106) in refractory solid tumors: safety, clinical activity, pharmacodynamics, and immunologic correlates. $\mathcal{f}$ Clin Oncol 28: 3167-3175.

Brahmer, J., Horn, L. and Antonia, S. (2013) Nivolumab (anti-PD-1; BMS-936558; ONO- 4538) in patients with non-small cell lung cancer (NSCLC): overall survival and long-term safety in a phase 1 trial. In: IASLC 15th World Conference on Lung Cancer, Sydney, Australia, MO18.03.

Brahmer, J., Horn, L., Gandhi, L., Spigel, D., Antonia, S., Rizvi, N. et al. (2014) Nivolumab (anti-PD-1, BMS-936558, ONO-4538) in patients (Pts) with advanced non-small-cell lung cancer (NSCLC): survival and clinical activity by subgroup analysis. ASCO Meeting Abstracts 32: 8112 .

Brahmer, J. and Pardoll, D. (2013) Immune checkpoint inhibitors: making immunotherapy a reality for the treatment of lung cancer. Cancer Immunol Res 1: 85-91.

Brahmer, J., Tykodi, S., Chow, L., Hwu, W., Topalian, S., Hwu, P. et al. (2012) Safety and activity 
of anti-PD-L1 antibody in patients with advanced cancer. N Engl f Med 366: 2455-2465.

Bristol-Myers Squibb (2014) Phase 2 objective response rate and survival data for Opdivo (nivolumab) in heavily pre-treated advanced squamous cell non-small cell lung cancer. To be presented at the 2014 Chicago Multidisciplinary Symposium on Thoracic Oncology, press release available at: http://news.bms.com/press-release/ rd-news/phase-2-objective-response-rate-and-survivaldata-opdivo-nivolumab-heavily-pre

Chen, D., Irving, B. and Hodi, F. (2012) Molecular pathways: next-generation immunotherapy-nhibiting programmed death-ligand 1 and programmed death1. Clin Cancer Res 18: 6580-6587.

Chen, Y., Mu, C. and Huang, J. (2012) Clinical significance of programmed death-1 ligand-1 expression in patients with non-small cell lung cancer: a 5-year-follow-up study.Tumori 98: 751-755.

Chen, Y., Wang, L., Zhu, H., Li, X., Zhu, Y., Yin, Y. et al. (2013) Relationship between programmed death-ligand 1 and clinicopathological characteristics in non-small cell lung cancer patients. Chin Med Sci f 28: $147-151$.

Curran, M., Montalvo, W., Yagita, H. and Allison, J. (2010) PD-1 and CTLA-4 combination blockade expands infiltrating $\mathrm{T}$ cells and reduces regulatory $\mathrm{T}$ and myeloid cells within B16 melanoma tumors. Proc Natl Acad Sci U S A 107: 4275-4280.

Dong, H., Strome, S., Salomao, D., Tamura, H., Hirano, F., Flies, D. et al. (2002) Tumor-associated B7-H1 promotes T-cell apoptosis: a potential mechanism of immune evasion. Nat Med 8: 793-800.

Downey, S., Klapper, J., Smith, F., Yang, J., Sherry, R., Royal, R. et al. (2007) Prognostic factors related to clinical response in patients with metastatic melanoma treated by CTL-associated antigen- 4 blockade. Clin Cancer Res 13: 6681-6688.

Einhorn, L., Bond, W., Hornback, N. and Joe, B. (1978) Long-term results in combined-modality treatment of small cell carcinoma of the lung. Semin Oncol 5: 309-313.

Fecher, L., Agarwala, S., Hodi, F. and Weber, J. (2013) Ipilimumab and its toxicities: a multidisciplinary approach. Oncologist 18: 733-743.

Formenti, S. and Demaria, S. (2013) Combining radiotherapy and cancer immunotherapy: a paradigm shift. F Natl Cancer Inst 105: 256-265.

Freire, J., Ajona, D., De Biurrun, G., Agorreta, J., Segura, V., Guruceaga, E. et al. (2013) Silica-induced chronic inflammation promotes lung carcinogenesis in the context of an immunosuppressive microenvironment. Neoplasia 15: 913-924.
Frey, B., Rubner, Y., Kulzer, L., Werthmöller, N., Weiss, E., Fietkau, R. et al. (2014) Antitumor immune responses induced by ionizing irradiation and further immune stimulation. Cancer Immunol Immunother 63: 29-36.

Gandhi, M., Lambley, E., Duraiswamy, J., Dua, U., Smith, C., Elliott, S. et al. (2006) Expression of LAG-3 by tumor-infiltrating lymphocytes is coincident with the suppression of latent membrane antigen-specific CD8 + T-cell function in Hodgkin lymphoma patients. Blood 108: 2280-2289.

Garon, E., Balmanoukian, A. and Hamid, O. (2013) Preliminary clinical safety and activity of MK-3475 mono- therapy for the treatment of previously treated patients with non-small cell lung cancer. In: IASLC 15th World Conference on Lung Cancer, Sydney, Australia.

Garon, E., Gandhi, L., Rizvi, N., Hui, R., Balmanoukian, A., Patnaik, A. et al. (2014a) Antitumor activity of pembrolizumab (Pembro; MK-3475) and correlation with programmed death ligand 1 (PD-L1) expression in a pooled analysis of patients with advanced NSCLC. ESMO Meeting Abstracts LBA43.

Garon, E., Leighl, N., Rizvi, N., Blumenschein, G., Balmanoukian, A., Eder, J. et al. (2014b) Safety and clinical activity of MK-3475 in previously treated patients (Pts) with non-small cell lung cancer (NSCLC). ASCO Meeting Abstracts 32: 8020.

Gettinger, S., Shepherd, F., Antonia, S., Brahmer, J., Chow, L., Juergens, R. et al. (2014) First-line nivolumab (anti-PD-1; BMS-936558, ONO-4538) monotherapy in advanced NSCLC: safety, efficacy, and correlation of outcomes with PD-L1 status. ASCO Meeting Abstracts 32: 8024.

Ghiringhelli, F., Menard, C., Puig, P., Ladoire, S., Roux, S., Martin, F. et al. (2007) Metronomic cyclophosphamide regimen selectively depletes $\mathrm{CD} 4+\mathrm{CD} 25+$ regulatory $\mathrm{T}$ cells and restores $\mathrm{T}$ and $\mathrm{NK}$ effector functions in end stage cancer patients. Cancer Immunol Immunother 56: 641-648.

Goel, S., Duda, D., Xu, L., Munn, L., Boucher, Y., Fukumura, D. et al. (2011) Normalization of the vasculature for treatment of cancer and other diseases. Physiol Rev 91: 1071-1121.

Grande, C., Villanueva, M., Huidobro, G. and Casal, J. (2007) Docetaxel-induced interstitial pneumonitis following non-small-cell lung cancer treatment. Clin Transl Oncol 9: 578-581.

Hamid, O., Robert, C., Daud, A., Hodi, F., Hwu, W., Kefford, R. et al. (2013) Safety and tumor responses with lambrolizumab (anti-PD-1) in melanoma. $N$ Engl f Med 369: 134-144.

Harmankaya, K., Erasim, C., Koelblinger, C., Ibrahim, R., Hoos, A., Pehamberger, H. et al. (2011) 
Continuous systemic corticosteroids do not affect the ongoing regression of metastatic melanoma for more than two years following ipilimumab therapy. Med Oncol 28: 1140-1144.

Harvey, R. (2014) Immunologic and clinical effects of targeting PD-1 in lung cancer. Clin Pharmacol Ther 96: 214-223.

Horn, L., Herbst, R., Spigel, D., Gettinger, S., Gordon, M., Hollebecque, A. et al. (2013) An analysis of the relationship of clinical activity to baseline EGFR status, PD-L1 expression and prior treatment history in patients with non-small cell lung cancer (NSCLC) following PD-L1 blockade with MPDL3280A (anti-PD-L1). In: 15th World Conference on Lung Cancer, Sydney, Australia.

Howlader, N., Noone, A., Krapcho, M., Garshell, J., Miller, D., Altekruse, S. et al. (2014) SEER Cancer Statistics Review, 1975-2011. Bethesda, MD: National Cancer Institute. Based on November 2013 SEER data submission, posted to the SEER web site, April 2014 http://seer.cancer.gov/csr/1975_2011/ (accessed 3 December 2014).

Huang, C., Workman, C., Flies, D., Pan, X., Marson, A., Zhou, G. et al. (2004) Role of LAG-3 in regulatory $\mathrm{T}$ cells. Immunity 21: 503-513.

Huang, Y., Goel, S., Duda, D., Fukumura, D. and Jain, R. (2013) Vascular normalization as an emerging strategy to enhance cancer immunotherapy. Cancer Res 73: 2943-2948.

Jansen, R., Slingerland, R., Goey, S., Franks, C., Bolhuis, R. and Stoter, G. (1992) Interleukin-2 and interferon-alpha in the treatment of patients with advanced non-small-cell lung cancer. F Immunother 12: $70-73$.

Jemal, A., Bray, F., Center, M., Ferlay, J., Ward, E. and Forman, D. (2011) Global cancer statistics. $C A$ 61: 69-90.

Keir, M., Butte, M., Freeman, G. and Sharpe, A. (2008) PD-1 and its ligands in tolerance and immunity. Annu Rev Immunol 26: 677-704.

Kodumudi, K., Woan, K., Gilvary, D., Sahakian, E., Wei, S. and Djeu, J. (2010) A novel chemoimmunomodulating property of docetaxel: suppression of myeloid-derived suppressor cells in tumor bearers. Clin Cancer Res 16: 4583-4594.

Konishi, J., Yamazaki, K., Azuma, M., Kinoshita, I., Dosaka-Akita, H. and Nishimura, M. (2004) B7-H1 expression on non-small cell lung cancer cells and its relationship with tumor-infiltrating lymphocytes and their PD-1 expression. Clin Cancer Res 10: 5094-5100.

Konishi, J., Yamazaki, K., Kinoshita, I., Isobe, H., Ogura, S., Sekine, S. et al. (2005) Analysis of the response and toxicity to gefitinib of non-small cell lung cancer. Anticancer Res 25: 435-441.
Kroemer, G., Galluzzi, L., Kepp, O. and Zitvogel, L. (2013) Immunogenic cell death in cancer therapy. Annu Rev Immunol 31: 51-72.

Leow, P., Soo, R., Soong, R. and Ou, S. (2012) Targeting ROS1 with anaplastic lymphoma kinase (ALK) inhibitors: a promising therapeutic strategy for a newly defined molecular subset of non-small cell lung cancer. F Thorac Oncol 7: 1625-1630.

Liu, W., Fowler, D., Smith, P. and Dalgleish, A. (2010) Pre-treatment with chemotherapy can enhance the antigenicity and immunogenicity of tumours by promoting adaptive immune responses. $\mathrm{Br} \mathcal{F}$ Cancer 102: 115-123.

Lutzky, J., Antonia, S., Blake-Haskins, A., Li, X., Robbins, P., Shalabi, A. et al. (2014) A phase 1 study of MEDI4736, an anti-PD-L1 antibody, in patients with advanced solid tumors. ASCO Meeting Abstracts 32: 3001 .

Lynch, T., Bondarenko, I., Luft, A., Serwatowski, P., Barlesi, F., Chacko, R. et al. (2012) Ipilimumab in combination with paclitaxel and carboplatin as first-line treatment in stage IIIb/IV non-small-cell lung cancer: results from a randomized, double-blind, multicenter phase II study. F Clin Oncol 30: 2046-2054.

Mu, C., Huang, J., Chen, Y., Chen, C. and Zhang, X. (2011) High expression of PD-L1 in lung cancer may contribute to poor prognosis and tumor cells immune escape through suppressing tumor infiltrating dendritic cells maturation. Med Oncol 28: 682-688.

ONO Pharmaceutical (2014) Human Anti-human PD-1 Monoclonal Antibody “OPDIVO ${ }^{\circledR}$ Intravenous Infusion 20 mg/100 mg” Receives Manufacturing and Marketing Approval in Japan for the Treatment of Unresectable Melanoma. Available at: http://www. ono.co.jp/eng/news/pdf/sm_cn140704.pdf (accessed 6 August 2014)

Oxnard, G., Morris, M., Hodi, F., Baker, L., Kris, M., Venook, A. et al. (2012) When progressive disease does not mean treatment failure: reconsidering the criteria for progression. F Natl Cancer Inst 104: 1534-1541.

Pardoll, D. (2012) The blockade of immune checkpoints in cancer immunotherapy. Nat Rev Cancer 12: 252-264.

Ramalingam, S., Mazieres, J., Planchard, D., Stinchcombe, T., Dy, G., Antonia, S. et al. (2014) Phase II study of nivolumab (Anti-PD-1, BMS936558, ONO-4538) in patients with advanced, refractory squamous non-small cell lung cancer. In: Multidisciplinary Symposium in Thoracic Oncology, abstract LB2.

Rizvi, N., Chow, L., Borghaei, H., Shen, Y., Harbison, C., Alaparthy, S. et al. (2014) Safety and response with nivolumab (anti-PD-1; BMS-936558, ONO4538) plus erlotinib in patients (Pts) with epidermal 
growth factor receptor mutant (EGFR $\mathrm{Mt}$ ) advanced NSCLC. ASCO Meeting Abstracts 32: 8022.

Romagne, F., Andre, P., Spee, P., Zahn, S., Anfossi, N., Gauthier, L. et al. (2009) Preclinical characterization of 1-7f9, a novel human anti-KIR receptor therapeutic antibody that augments natural killer-mediated killing of tumor cells. Blood 114 : 2667-2677.

Rozali, E., Hato, S., Robinson, B., Lake, R. and Lesterhuis, W. (2012) Programmed death ligand 2 in cancer-induced immune suppression. Clin Dev Immunol 2012: 656340.

Ruggeri, L., Capanni, M., Urbani, E., Perruccio, K., Shlomchik, W., Tosti, A. et al. (2002) Effectiveness of donor natural killer cell alloreactivity in mismatched hematopoietic transplants. Science 295: 2097-2100.

Schiller, J., Morgan-Ihrig, C. and Levitt, M. (1995) Concomitant administration of interleukin-2 plus tumor necrosis factor in advanced non-small cell lung cancer. Am f Clin Oncol 18: 47-51.

Schreiber, R., Old, L. and Smyth, M. (2011) Cancer immunoediting: integrating immunity's roles in cancer suppression and promotion. Science 331: 1565-1570.

Soria, J., Cruz, C., Bahleda, R., Delord, J., Horn, L., Herbst, R. et al. (2013) Clinical activity, safety and biomarkers of PD-L1 blockade in non-small cell lung cancer (NSCLC): additional analyses from a clinical study of the engineered antibody MPDL3280A (antiPD-L1). In: European Cancer Congress, Amsterdam, The Netherlands, abstract 3408.

Stewart, T. and Abrams, S. (2007) Altered immune function during long-term host-tumor interactions can be modulated to retard autochthonous neoplastic growth. F Immunol 179: 2851-2859.

Solomon, B., Varella-Garcia, M. and Camidge, D. (2009) ALK gene rearrangements: a new therapeutic target in a molecularly defined subset of non-small cell lung cancer. F Thorac Oncol 4: 1450-1454.

Sorensen, S., Zhou, W., Dolled-Filhart, M., Georgsen, J., Wang, Z., Emancipator, K. et al. (2014) PD-L1 expression and survival among advanced nonsmall cell lung cancer (NSCLC) patients treated with chemotherapy. In: European Cancer Congress Meeting Abstracts, 1328P.

Sundar, R., Soong, R., Cho, B., Brahmer, J. and Soo, R. (2014) Immunotherapy in the treatment of nonsmall cell lung cancer. Lung Cancer 85: 101-109.

Visit SAGE journals online http://tam.sagepub.com

@SAGE journals
Suzuki, E., Kapoor, V., Jassar, A., Kaiser, L. and Albelda, S. (2005) Gemcitabine selectively eliminates splenic Gr-1+/CD11b+ myeloid suppressor cells in tumor-bearing animals and enhances antitumor immune activity. Clin Cancer Res 11: 6713-6721.

Taube, J., Anders, R., Young, G., Xu, H., Sharma, R., McMiller, T. et al. (2012) Colocalization of inflammatory response with B7-H1 expression in human melanocytic lesions supports an adaptive resistance mechanism of immune escape. Sci Transl Med 4: 127-137.

Topalian, S., Hodi, F., Brahmer, J., Gettinger, S., Smith, D., McDermott, D. et al. (2012) Safety, activity, and immune correlates of anti-PD-1 antibody in cancer. $N$ Engl F Med 366: 2443-2454.

Tseng, S., Otsuji, M., Gorski, K., Huang, X., Slansky, J., Pai, S. et al. (2001) B7-DC, a new dendritic cell molecule with potent costimulatory properties for $\mathrm{T}$ cells. F Exp Med 193: 839-846.

Van Den Eynde, B. and Boon, T. (1997) Tumor antigens recognized by $\mathrm{T}$ lymphocytes. Int $\mathcal{F}$ Clin Lab Res 27: 81-86.

Velcheti, V., Schalper, K., Carvajal, D., Anagnostou, V., Syrigos, K., Sznol, M. et al. (2014) Programmed death ligand-1 expression in non-small cell lung cancer. Lab Invest 94: 107-116.

Wrangle, J., Wang, W., Koch, A., Easwaran, H., Mohammad, H., Vendetti, F. et al. (2013) Alterations of immune response of non-small cell lung cancer with azacytidine. Oncotarget 4: 2067-2079.

Wolchok, J., Hoos, A., O’Day, S., Weber, J., Hamid, O., Lebbe, C. et al. (2009) Guidelines for the evaluation of immune therapy activity in solid tumors: immune-related response criteria. Clin Cancer Res 15: 7412-7420.

Wolchok, J., Kluger, H., Callahan, M., Postow, M., Rizvi, N., Lesokhin, A. et al. (2013) Nivolumab plus ipilimumab in advanced melanoma. $N$ Engl $\mathcal{F ~ M e d ~}$ 369: 122-133.

Yang, C., Lin, M., Chang, Y., Wu, C. and Yang, P. (2014) Programmed cell death-ligand 1 expression in surgically resected stage I pulmonary adenocarcinoma and its correlation with driver mutations and clinical outcomes. Eur f Cancer 50: 1361-1369.

Zhang, L., Dermawan, K., Jin, M., Liu, R., Zheng, H., Xu, L. et al. (2008) Differential impairment of regulatory $\mathrm{T}$ cells rather than effector $\mathrm{T}$ cells by paclitaxel-based chemotherapy. Clin Immunol 129: 219-229.

Zhang, Y., Wang, L., Li, Y., Pan, Y., Wang, R., Hu, H. et al. (2014) Protein expression of programmed death 1 ligand 1 and ligand 2 independently predict poor prognosis in surgically resected lung adenocarcinoma. Onco Targets Ther 7: 567-573. 